\title{
The Number of Compatible Pair of Actions for Cyclic Groups of 2-Power Order
}

\author{
Sahimel Azwal Sulaiman ${ }^{\text {a }}$, Mohd Sham Mohamad ${ }^{\mathrm{b}}$, Yuhani Yusof ${ }^{\mathrm{c}}$, and Mohammed Khalid Shahoodh ${ }^{\mathrm{d}}$ \\ Applied \& Industrial Mathematics (AIMs) Research Cluster, Faculty of Industrial Sciences \& Technology \\ Universiti Malaysia Pahang, Kuantan, Malaysia. \\ a pse13002@stdmail.ump.edu.my, ${ }^{\mathrm{b}}$ mohdsham@ump.edu.my, ${ }^{\mathrm{c}}$ yuhani@ump.edu.my, ${ }^{\mathrm{d}}$ moha861122@yahoo.com
}

\begin{abstract}
The non-abelian tensor product of groups has its origins in the algebraic K-theory and homotopy theory. The nonabelian tensor product for a pair of groups is defined when the actions act compatibly on each other. This research is to determine the maximum number of a compatible pair of actions that can be identified between two cyclic groups of 2-power order for nonabelian tensor product. The compatible pair of actions between two cyclic groups of 2-power order can be found by using the necessary and sufficient conditions of two cyclic groups of 2-power order acting compatibly on each other. Hence, the number of the compatible pair of actions between two cyclic groups of the 2-power order is determined.
\end{abstract}

Keywords - Compatible Actions, Cyclic Groups, Non-abelian Tensor Product.

\section{INTRODUCTION}

The non-abelian tensor product of a pair of groups which act on each other has its origins in connection with a generalized Van Kampen Theorem. Its construction has its origins in the algebraic $K$-theory and also homotopy theory. Let $G$ and $H$ be groups, then the non-abelian tensor product, $G \otimes H$ is the group generated by $g \otimes h$ and satisfy two relations:

$$
g g^{\prime} \otimes h=\left({ }^{g} g^{\prime} \otimes{ }^{g} h\right)(g \otimes h)
$$

And

$$
g \otimes h h^{\prime}=(g \otimes h)\left({ }^{h} g \otimes{ }^{h} h^{\prime}\right)
$$

The concept of the non-abelian tensor product of groups with compatible actions introduced by Brown and Loday (1984) is a continuation from the idea by Whitehead (1950). If $G$ and $H$ act on each other and each of which acts on itself by conjugation and satisfies ${ }^{\left({ }^{g} h\right)} g^{\prime}={ }^{g}\left({ }^{h}\left(g^{-1} g^{\prime}\right)\right)$ and ${ }^{\left({ }^{h} g\right)} h^{\prime}={ }^{h}\left({ }^{g}\left({ }^{h^{-1}} h^{\prime}\right)\right)$, then the actions are said to be compatible.

Many researchers have studied group theoretical aspect on non-abelian tensor product to answer some open problems given by Brown et. al. (1987). Kappe (1999) shows some overview of known results and literature on non-abelian tensor product up to 1997 . There are many researchers working on non-abelian tensor square $G \otimes G$ and defined the actions as trivial for both actions. If $G \neq H$, then the compatibility needs to be checked.

McDermott (1998) had determined the order of the non-abelian tensor product for all normal subgroup $G$ and $H$ by using Groups, Algorithms and Programming (GAP) software and divided into two cases. The first case for quaternion group of order 32 and the second case when the quaternion group and dihedral group of order 8. Ellis and McDermott (1998) checked the compatible conditions on computing the non-abelian tensor product for different groups and the actions are nontrivial. They focus on quaternion groups of order up to 32 only. Visscher (1998) worked on cyclic groups of $p$-power order where $p$ is prime and provided necessary and sufficient conditions for a pair of cyclic groups to act compatibly on each other. He also showed the compatibility when one of the actions is trivial and when both actions are trivial. Next, Mohamad in 2012 gave the new necessary and sufficient conditions and the characterization that pair of cyclic groups of a $p$ power order act compatibly on each other where the conditions are depended on the order of the actions.

A paper by Mohamad et. al (2012) gave the compatibility conditions and the non-abelian tensor product of the cyclic group of order $p^{2}$ with the actions of order $p$. While Sulaiman et al. (2015), investigated some compatible pairs of nontrivial actions of order two and four for some cyclic groups of 2-power order. Next, Sulaiman et al. (2016) investigated the compatible pair of nontrivial action for finite cyclic 2-groups when two groups are same and the actions are the same order. Lastly, Shahoodh et al. (2016) studied the compatible pair of actions for two cyclic groups of 3-power order. In this paper, the main concern is on determining the maximum number of the compatible pair of actions can be identified between two cyclic groups of 2-power order.

In Section 2, some preparatory definitions, and results for the compatible pairs of actions are given. While in Section 3, the number of the compatible pair of actions for cyclic groups of 2-power order is determined. Next, in Section 4 some examples regarding results for the number of the compatible pair of actions for cyclic groups of 2- 
power order are presented. Lastly, the conclusions for this paper are given in section 5 .

\section{SOME PREPARATORY ON COMPATIBLE CONDITIONS}

In this section, some definitions and previous results on compatible conditions that will be used in proving the new results will be stated. The definition of a compatible pair of actions between two groups by Visscher (1998) will first be given as below.

\subsection{Definition}

Let $G$ and $H$ be groups which act on each other. These mutual action are said to be compatible with each other and with the actions of $G$ and $H$ on themselves by conjugation if:

$$
\begin{aligned}
& { }^{\left({ }^{g} h\right)} g^{\prime}={ }^{g}\left({ }^{h}\left({ }^{g^{-1}} g^{\prime}\right)\right) \\
& \text { and } \\
& { }^{\left({ }^{h} g\right)} h^{\prime}={ }^{h}\left({ }^{g}\left({ }^{h^{-1}} h^{\prime}\right)\right) \\
& \text { for all } g, g^{\prime} \in G \text { and } h, h^{\prime} \in H .
\end{aligned}
$$

In this paper, the cyclic groups are the consideration groups. Let $G$ and $H$ are two finite cyclic groups, and then the action of $G$ and $H$ is required to have the property that the identity in $G$ acts as the identity mapping on $H$. Thus, all elements in $G$ act as auto-morphism $H$ on $H$. In the case $G$ is an abelian group, Visscher (1998) in Corollary 2.2 shows that every pair of actions is compatible when one of the actions is trivial.

\subsection{Corollary}

Let $G$ and $H$ be groups. Furthermore, let $G$ act trivially on $H$. If $G$ is abelian, then for any action of $H$ on $G$ the mutual actions are compatible.

Next, Dummit and Foote (2004) shows that the automorphism of a cyclic group is isomorphic with a direct product of two cyclic groups as stated in following:

\subsection{Theorem}

Let $G$ be a cyclic group of order $2^{n}, n \geq 3$.

$$
\begin{aligned}
& \text { Then, } \operatorname{Aut}(G) \cong C_{2} \times C_{2^{n-2}} \\
& \text { and } \\
& \operatorname{Aut}(G) \mid=\varphi\left(2^{n}\right)=2^{n-1} .
\end{aligned}
$$

Mohamad (2012) characterized for every automorphisms of a finite cyclic group of 2-power order. He represented the generator for automorphism of a finite cyclic group of 2-power order and expressed every auto-morphism in terms of generators, $\tau$ and $\rho$. The following theorem gives the representation of auto-morphisms group for a finite cyclic group of 2-power order.

2.4 Theorem

Let $\quad G=\langle g\rangle \cong C_{2^{n}}, n \geq 3 . \quad$ Then, $\operatorname{Aut}(G)=\langle\tau\rangle \times\langle\rho\rangle, \quad$ where $\quad \tau(g)=g^{-1} \quad$ and $\rho(g)=g^{5}$ and every $\sigma \in \operatorname{Aut}(G)$ can be represented as $\sigma=\tau^{i} \rho^{i}$ with $i=0,1$ and $j=0,1, \ldots, 2^{n-2}-1$ and $\tau^{i} \rho^{i}(g)=g^{t}$ with $t \equiv(-1)^{i} \cdot 5^{j} \bmod 2^{n}$.

Next, the number of auto-morphisms for two cyclic groups of 2-power order with a specific order done by Mohamad (2012) in given the following corollary.

\subsection{Corollary}

Let $G=\langle g\rangle \cong C_{2^{n}}, n \geq 3$.

Then, Aut $(G)=\langle\tau\rangle \times\langle\rho\rangle$, where $\tau(g)=g^{-1}$ and $\rho(g)=g^{5}$. Then there exist three auto-morphisms of order two, namely $\sigma=\tau, \rho^{2^{n-3}}$ and $\tau \rho^{2^{n-3}}$ with $\tau(g)=g^{-1}, \rho^{2^{n-3}}(g)=g^{5^{j}} \quad$ and $\tau \rho^{2^{n-3}}=g^{-5^{j}}$, where $j=2^{n-3}$. Furthermore, there exist $2^{s}$ automorphisms of order $2^{s}, s=2,3, \ldots, n-2$ namely $\sigma=\rho^{j}, \tau \rho^{j}$ with $\rho^{j}(g)=g^{5^{j}}$ and $\tau \rho^{j}(g)=g^{-5^{j}}$ where $\operatorname{gcd}\left(2^{n-2}, j\right)=2^{n-s-2}$.

According to the presentation of auto-morphisms group for two cyclic groups of 2-power order, Mohamad (2012) did the characterization of every compatible pair of actions. He represented the necessary and sufficient condition for the compatible pair of actions for any two auto-morphisms for the specific order. The compatible conditions when the actions have an order two are given in the following theorem.

\subsection{Theorem}

Let $\quad G=\langle x\rangle \cong C_{2^{m}} \quad$ and $\quad H=\langle y\rangle \cong C_{2^{n}}$. Furthermore, let $\sigma \in \operatorname{Aut}(G)$ with $|\sigma|=2$ and $\sigma^{\prime} \in \operatorname{Aut}(H)$, where $m \geq 1, n \geq 3$.

i. If $\sigma(x)=x^{t}$ with $t \equiv-1 \bmod 2^{m}$ or $t \equiv 2^{m-1}-1 \bmod 2^{m}$, then $\left(\sigma, \sigma^{\prime}\right)$ is a compatible pair if and only if the trivial auto-morphism or $\left|\sigma^{\prime}\right|=2$. 
ii. If $G=\langle x\rangle \cong C_{2^{m}}$ with $t \equiv 2^{m-1}+1 \bmod 2^{m}$, then $\left(\sigma, \sigma^{\prime}\right)$ is compatible pair if and only if $\left|\sigma^{\prime}\right|=2^{s^{\prime}}$ with $s^{\prime} \leq m-1$ in particular $\sigma$ is compatible with all $\sigma^{\prime} \in \operatorname{Aut}(H)$ provided $n \leq m+1$.

Next, Mohamad (2012) gives the characterization of the compatible conditions when the actions have an order greater than two are stated as follow.

\subsection{Theorem}

Let $\quad G=\langle x\rangle \cong C_{2^{m}} \quad$ and $\quad H=\langle y\rangle \cong C_{2^{n}}$. Furthermore, let $\sigma \in \operatorname{Aut}(G)$ with $|\sigma|=2^{s}, s \geq 2$ and $\sigma^{\prime} \in \operatorname{Aut}(H)$, where $m \geq 4, n \geq 1$.

i. If $\sigma(x)=x^{t}$ with $t \equiv(-1)^{i} 5^{j} \bmod 2^{m}$ and $i=1$, then $\left(\sigma, \sigma^{\prime}\right)$ is a compatible pair if and only if $\sigma^{\prime}(y)=y^{t^{\prime}}$ with $t^{\prime} \equiv 1 \bmod 2^{n}$ and $t^{\prime} \equiv 2^{n-1}+1 \bmod$ $2^{n}$.

ii. If $\sigma(x)=x^{t}$ with $t \equiv(-1)^{i} 5^{j} \bmod 2^{m}$ and $i=0$, then $\left(\sigma, \sigma^{\prime}\right)$ is a compatible pair if and only if $\left|\sigma^{\prime}\right| \leq 2^{m-s}$ provided $n \leq m-s+2$.

In 2016, Sulaiman et. al represent the necessary and sufficient conditions for two cyclic groups of 2-power order that compatibly act on each other for the case of $G$ $=H$. The compatible conditions with the same order of actions are given in the following proposition.

\subsection{Proposition}

Let $G=H=\langle g\rangle @ C_{2^{m}}$. Furthermore, let $\sigma \in$ $\operatorname{Aut}(G)$ and $\sigma^{\prime} \in \operatorname{Aut}(H)$ with $|\sigma|=\left|\sigma^{\prime}\right|=2^{k} .\left(\sigma, \sigma^{\prime}\right)$ is compatible pair of actions if $k=0, k=1$ and $k^{3} 2$ with $\sigma(g)=g^{t}$ where $t \equiv 5^{j} \bmod 2^{m}$.

Meanwhile, Sulaiman et al. (2016) also gives the exact number of the compatible pair of nontrivial actions for two cyclic groups of 2-power order with the same order of actions.

\subsection{Proposition}

Let $G=H=\langle g\rangle @ C_{2^{m}}$. Furthermore, let $\sigma \in$ $\operatorname{Aut}(G)$ and $\sigma^{\prime} \in \operatorname{Aut}(H)$ with $|\sigma|=\left|\sigma^{\prime}\right|=2^{k}$. Then,

i. if $k=0$, there is one compatible pair of actions.

ii. if $k=1$, there are 9 compatible pair of actions. iii. if $k \geq 2$ there are $2^{2 k-2}$ compatible pair of actions.

Extended from the paper by Sulaiman et al. (2016), the results for the numbers of the compatible pair of actions for two cyclic groups of 2-power order are given in the following section.

\section{RESULTS AND DISCUSSION}

In this section, the number of the compatible pair of actions can be determined by using the necessary and sufficient conditions for two cyclic groups of 2-power order to act compatibly on each other. First, let one of the actions has an order one, then the number of the compatible pair of actions is given as follows.

\subsection{Corollary}

Let $G \cong C_{2^{m}}$ and $H \cong C_{2^{n}}$. Furthermore, let $\sigma \in$ $\operatorname{Aut}(G)$ with $|\sigma|=1$ and $\sigma^{\prime} \in \operatorname{Aut}(H)$, where $m \geq 1, n \geq 1$. The number of the compatible pair of actions is $2^{n-1}$.

Proof Let $G \cong C_{2^{m}}$ and $H \cong C_{2^{n}}$. Furthermore, let $\sigma \in \operatorname{Aut}(G)$ with $|\sigma|=1$ and $\quad \sigma^{\prime} \in \operatorname{Aut}(H)$, where $m \geq 1, n \geq 1$. By Corollary 2.2, when $G$ act trivially on $H$, then the mutual actions of any action $H$ on $G$ are compatible. Thus, by Theorem 2.3, the number of the compatible pair of actions is given as $2^{n-1}$ since | $\operatorname{Aut}(H) \mid=2^{n-1}$.

Next, the compatible pair of actions where one of the actions has an order two is determined. By Theorem 2.6, there are two cases for the compatible pair of actions has an order two. The first case is $\sigma(x)=x^{t}$ with $t \equiv-1$ $\bmod 2^{m}$ or $t \equiv 2^{m-2}-1 \bmod 2^{m}$ and the second case is $\sigma(x)=x^{t}$ with $t \equiv 2^{m-2}+1 \bmod 2^{m}$. The following proposition will give the number of the compatible pair of actions where one of the actions has an order two.

\subsection{Proposition}

Let $G \cong C_{2^{m}}$ and $H \cong C_{2^{n}}$. Furthermore, let $\sigma \in \operatorname{Aut}(G) \quad$ with $\quad|\sigma|=2$ and $\sigma^{\prime} \in \operatorname{Aut}(H)$, where $m \geq 1, n \geq 3$.

$$
\text { i. If } \sigma(x)=x^{t} \text { with } t \equiv-1 \bmod 2^{m} \text { or }
$$
$t \equiv 2^{m-1}-1 \bmod 2^{m}$, then there are eight compatible pairs of actions. 
ii. If $G=\langle x\rangle \cong C_{2^{m}}$ with $t \equiv 2^{m-1}+1 \bmod 2^{m}$, then there are $2^{r-1}$ compatible pairs of actions where $r=\min \{n, m+1\}$.

Proof: Let $G \cong C_{2^{m}}$ and $H \cong C_{2^{n}}$. Furthermore, let $\sigma \in \operatorname{Aut}(G)$ with $|\sigma|=2$ and $\sigma^{\prime} \in \operatorname{Aut}(H)$, where $m \geq 1, n \geq 3$.

i. $\quad$ Let $t \equiv-1 \bmod 2^{m}$ or $t \equiv 2^{m-1}-1 \bmod 2^{m}$. First, consider $\left|\sigma^{\prime}\right|=1$ and its only one auto-morphism under this consideration which is trivial action. Thus, two compatible pairs of actions are exits. Next, consider $\left|\sigma^{\prime}\right|=2$, then by Theorem 2.6(i), the actions are always compatible with all actions having order two. Thus, there are six compatible pairs under this case. Therefore, if $\sigma(x)=x^{t}$ with $t \equiv-1 \bmod 2^{m}$ or $t \equiv 2^{m-1}-1 \bmod$ $2^{m}$, then there are eight compatible pairs of actions.

ii. Let $t \equiv 2^{m-1}+1 \bmod 2^{m}$. By Theorem 2.3, there are $2^{n-1}$ auto-morphism in $\operatorname{Aut}(H)$. Thus, by Theorem 2.6(ii) follows that the number of the compatible pair of actions is $2^{r-1}$ where $r=\min \{n, m+1\}$.

In particular, the number of the compatible pair of actions has an order two is presented in the next theorem.

3.3 Theorem

Let $G \cong C_{2^{m}}$ and $H \cong C_{2^{n}}$. Furthermore, let $\sigma \in$ $\operatorname{Aut}(G)$ and $\sigma^{\prime} \in \operatorname{Aut}(H)$. If $|\sigma|=2$, then there are $2^{r-1}+8$ number of the compatible pair of actions for $G \otimes H$ with $r=\min \{n, m+1\}$.

Proof Let $G \cong C_{2^{m}}$ and $H \cong C_{2^{n}}$. Furthermore, let $\sigma \in \operatorname{Aut}(G) \quad$ and $\quad \sigma^{\prime} \in \operatorname{Aut}(H)$. If $|\sigma|=2$. By Proposition 3.2, there are $2^{r-1}+8$ number of the compatible pair of actions for $G \otimes H$ with $r=\min \{n, m+1\}$.

Now, the number of the compatible pair of actions when one of the actions has an order greater than two are determined. By Theorem 2.7, there are two cases for the compatible conditions for any two auto-morphisms with a specific order. The first case is $\sigma(g)=g^{t}$ with $t \equiv(-1)^{i} 5^{j} \bmod 2^{m}$ and $i=1$, while the second case is $\sigma(g)=g^{t}$ with $t \equiv(-1)^{i} 5^{j} \bmod 2^{m}$ and $i=0$. Thus, to find the number of compatible pair of actions, two cases which $i=1$ and $i=0$ are need to be considered. Lemma 3.4 and Lemma 3.5 shows the number of compatible pair of actions for $i=1$ and $i=0$, respectively.

\subsection{Lemma}

Let $G \cong C_{2^{m}}$ and $H \cong C_{2^{n}}$. Furthermore, let $\sigma \in$ $\operatorname{Aut}(G)$ with $|\sigma|=2^{s}, s \geq 2$ and $\quad \sigma^{\prime} \in$ $\operatorname{Aut}(H)$, where $m \geq 4, n \geq 1$. If $\sigma(g)=g^{t}$ with $t \equiv(-1)^{i} 5^{j} \bmod 2^{m}$ and $i=1$, then there are $2^{s}$ compatible pair of actions.

Proof Let $G \cong C_{2^{m}}$ and $H \cong C_{2^{n}}$. Furthermore, let $\sigma \in \quad \operatorname{Aut}(G) \quad$ with $\quad|\sigma|=2^{s}, s \geq 2 \quad$ and $\sigma^{\prime} \in \operatorname{Aut}(H)$, where $m \geq 4, n \geq 1$. By Corollary 2.5, there exist $2^{s}$ auto-morphisms of order $2^{s}$. Suppose that $\sigma(g)=g^{t}$ with $t \equiv(-1)^{i} 5^{j} \bmod 2^{m}$ and $i=1$. By Theorem 2.7(i), $\left(\sigma, \sigma^{\prime}\right)$ is a compatible pair when $\sigma^{\prime}(h)=h^{t^{\prime}}$ with $t^{\prime} \equiv 1 \bmod 2^{n}$ and $t^{\prime} \equiv 2^{n-1}+1 \bmod$ $2^{n}$ only and there exist $2^{s-1}$ number of auto-morphisms which is half from the total number of auto-morphisms of order $2^{s}$. Thus, the number of compatible pair of actions for both $t^{\prime}$ for $i=1$ is $2^{s-1}+2^{s-1}=2^{s}$.

\subsection{Lemma}

Let $G \cong C_{2^{m}}$ and $H \cong C_{2^{n}}$. Furthermore, let $\sigma \in \operatorname{Aut}(G) \quad$ with $\quad|\sigma|=2^{s}, s \geq 2 \quad$ and $\sigma^{\prime} \in \operatorname{Aut}(H)$, where $m \geq 4, n \geq 1$. If $\sigma(g)=g^{t}$ with $t \equiv(-1)^{i} 5^{j} \bmod 2^{m}$ and $i=0$, then there are $2^{r^{\prime}-1}$ compatible pair of actions with $r^{\prime}=\min \{m, n\}$.

Proof Let $G \cong C_{2^{m}}$ and $H \cong C_{2^{n}}$. Furthermore, let $\sigma \in \operatorname{Aut}(G) \quad$ with $\quad|\sigma|=2^{s}, s \geq 2 \quad$ and $\sigma^{\prime} \in \operatorname{Aut}(H)$, where $m \geq 4, n \geq 1$. Suppose that $\sigma(g)=g^{t}$ with $t \equiv(-1)^{i} 5^{j} \bmod 2^{m}$ and $i=0$. By Theorem 2.7, $\sigma$ is compatible with all $\sigma^{\prime}$ provided $s^{\prime} \leq n-2$ and $s^{\prime} \leq m-2$ or simply presented as $s^{\prime} \leq$ $\min \{n-s, m-s\}$. Suppose that $r^{\prime}=\min \{n, m\}$, hence all $\sigma^{\prime}$ where $\left|\sigma^{\prime}\right| \leq 2^{r^{\prime}-s}$ are compatible with $\sigma$ when $\left|\sigma^{\prime}\right|=2^{s}$. If all actions are consider have an order 
$2^{s}$

with

$s \geq 2$,

then

$2^{2}+2^{3}+\ldots+2^{r^{\prime}-s}=2^{r^{\prime}-s+1}-4$. By Theorem 2.7, half auto-morphisms are considered for $i=0$ which resulted as $\frac{2^{r^{\prime}-s+1}-4}{2}=2^{r^{\prime}-s}-2$.

By Theorem 2.7, $\sigma$ is compatible with trivial action and one action with $\left|\sigma^{\prime}\right|=2$. Thus, the number of the compatible pair of actions for $\sigma$ with specific order is $2^{r^{\prime}-s}-2+2=2^{r^{\prime}-s}$.

By Theorem 2.3, there are $2^{s-1}$ auto-morphisms are consider when $i=0$. Therefore, the number of the compatible pair of action for $|\sigma|=2^{s}$ are $2^{s-1}\left(2^{r^{\prime}-s}\right)=2^{r^{\prime}-1}$ where $r^{\prime}=\min \{m, n\}$.

In specifically, the following lemma gives the number of the compatible pair of actions when one of the actions has an order greater than two.

\subsection{Lemma}

Let $G \cong C_{2^{m}}$ and $H \cong C_{2^{n}}$. Furthermore, let $\sigma \in \operatorname{Aut}(G)$ with $|\sigma|=2^{s}, s \geq 2$ and $\sigma^{\prime} \in \operatorname{Aut}(H)$, where $m \geq 4, n \geq 1$. If $\left(\sigma, \sigma^{\prime}\right)$ be the compatible pair of actions, then the number of the compatible pair of actions is $\left(2^{m-1}-4\right)+(m-3)\left(2^{r^{\prime}-1}\right)$ provided $r^{\prime}=$ $\min \{m, n\}$.

Proof Let $G \cong C_{2^{m}}$ and $H \cong C_{2^{n}}$. Furthermore, let $\sigma \in \operatorname{Aut}(G)$ with $|\sigma|=2^{s}, s \geq 2$ and $\quad \sigma^{\prime} \in \operatorname{Aut}(H)$, where $m \geq 4, n \geq 1$. Lemma 3.4 and Lemma 3.5 show that the number of the compatible pair of actions when one of the actions has order greater than two can divides into two cases i.e $i=1$ and $i=0$.

i. Suppose that $i=1$. By Lemma 3.4, there are $2^{s}$ compatible pair of actions. Without loss of generality, consider the highest order of $\sigma$ in $C_{2^{m}}$ is $2^{m-2}$. Then, the number of the compatible pair of actions is $2^{2}+2^{3}+2^{4}+\ldots+2^{m-2}=2^{m-1}-4$.

ii. Suppose that $i=0$. By Lemma 3.5, there are $2^{r^{\prime}-1}$ compatible pair of actions. Note that, the highest order of $\sigma$ in $C_{2^{m}}$ is $2^{m-2}$. Then, the number of the compatible pair of actions for the actions have order $2^{s}$ where $s=2,3,4, \ldots, 2^{m-2} \quad$ are

$(m-3) 2^{r^{\prime}-1}$ provided $r^{\prime}=\min \{m, n\}$.

Thus, the number of the compatible pair of actions when one of the actions has an order greater than two for both cases given as $2^{m-1}-4+(m-3) 2^{r^{\prime}-1}$.

Up to the extended, the number of the compatible pair of actions of 2-power order for a given non-abelian tensor product, $C_{2^{m}} \otimes C_{2^{n}}$ can be found. The result stated in the following theorem.

3.7 Theorem

Let $G \cong C_{2^{m}}$ and $H \cong C_{2^{n}}$, where $m \geq 1, n \geq 1$. Then, there exist $(m-3)\left(2^{r^{\prime}-1}\right)+2^{r-1}+2^{n-1}+2^{m-1}+4$ compatible pair of actions where $r^{\prime}=\min \{m, n\}$.

Proof Let $G \cong C_{2^{m}}$ and $H \cong C_{2^{n}}$, where $m \geq 1, n \geq 1$. Corollary 3.1, Theorem 3.3 and Lemma 3.6 show that the number of the compatible pair of actions have an action with specific order is determined. There are three cases which are $|\sigma|=1,2$ and $2^{s}$ where $s \geq 2$. Thus, three cases are considered.

i. Suppose that $|\sigma|=1$. By Corollary 3.1, when one of the action is trivial, then the number of the compatible pair of actions are $2^{n-1}$.

ii. Suppose that $|\sigma|=2$. By Theorem 3.3, there are $2^{m-1}+8$ number of compatible pair of actions.

iii. Suppose that $|\sigma|=2^{s}$ where $s \geq 2$. By Lemma 3.6, the number of the compatible pair of actions has order greater than two are $2^{m-1}-4+(m-3) 2^{r^{\prime}-1}$ where $r^{\prime}=\min \{m, n\}$

Hence, the number of the compatible pair of actions for finite cyclic groups of 2-power order is:

$$
\begin{aligned}
& 2^{n-1}+2^{r-1}+8+2^{m-1}-4+(m-3)\left(2^{r^{\prime}-1}\right)= \\
& (m-3)\left(2^{r^{\prime}-1}\right)+2^{r-1}+2^{n-1}+2^{m-1}+4
\end{aligned}
$$

Now, the maximum number of a compatible pair of actions between two cyclic groups of 2-power order for non-abelian tensor product, $C_{2^{m}} \otimes C_{2^{n}}$ has identified. The cases are separated into three main cases. The first case covered when one of the actions has order one which give $2^{n-1}$ pair of actions. While the second case covered when one of the actions has order two that give $2^{m-1}+8$ 
pair of actions. Finally, the third case covered when one of the actions has order greater than two which give $2^{m-1}-4+(m-3) 2^{r^{\prime}-1}$ with $r^{\prime}=\min \{m, n\}$

In the next section, the example shows the number of the compatible pair of actions of 2-power order for $G \otimes H$ are not necessary equal with $H \otimes G$ when $G \neq H$.

\section{AN EXAMPLE IN THE NUMBER OF COMPATIBLE PAIR OF ACTIONS}

Let $G=C_{2^{4}}$ and $H=C_{2^{6}}$ be finite cyclic groups. Now, consider the action of $G$ and $H$ act on each other such that ${ }^{h} g=g^{k}$ and ${ }^{g} h=h^{l}$ for $g \in G, h \in H$ and $k, l \in \square$. Table 1 illustrates the compatible pair of actions for $C_{2^{4}} \otimes C_{2^{6}}$ given by GAP software. From the table, there are 68 compatible pair of actions for $C_{2^{4}} \otimes C_{2^{6}}$. By Theorem 3.7, the number of the compatible pair of actions is $(4-3)\left(2^{4-1}\right)+2^{5-1}+2^{6-1}+2^{4-1}+4=68 \quad$ since $r=\min \{4+1,6\}=5$ and $\quad r^{\prime}=\min \{4,6\}=4$. Hence the result from Theorem 3.7 is equivalent with the number of the compatible pair of actions given in Table 1.

Now, there exist an enquiry on finding the equality of the number of the compatible pair of actions of 2-power order for $G \otimes H$ is equal with $H \otimes G$ for the case of $G \neq H$. Table 2 show the number of the compatible pair of actions for $C_{2^{m}} \otimes C_{2^{n}}$ given by GAP software. Let $G=C_{2^{4}}$ and $H=C_{2^{6}}$. Table 1 and Table 2 show there are 68 number of the compatible pair of actions for $G \otimes H$ but only 76 for $H \otimes G$. Thus, the prove holds.

TABLE 1 COMPATIBLE PAIR OF ACTIONS FOR $C_{24} \otimes C$

\begin{tabular}{|c|c|c|c|c|c|c|c|c|c|c|c|}
\hline \\
\hline$g^{k}$ & $k$ & $l$ & $\mid h^{l}$ & $g^{k}$ & $k$ & $l$ & $h^{l}$ & $g^{k}$ & $k$ & $l$ & $h^{l}$ \\
\hline 1 & 1 & 1 & 1 & 1 & 1 & 47 & 4 & 2 & 9 & 17 & 4 \\
\hline 1 & 1 & 3 & 16 & 1 & 1 & 49 & 4 & 2 & 9 & 23 & 8 \\
\hline 1 & 1 & 5 & 16 & 1 & 1 & 51 & 16 & 2 & 9 & 25 & 8 \\
\hline 1 & 1 & 7 & 8 & 1 & 1 & 53 & 16 & 2 & 9 & 31 & 2 \\
\hline 1 & 1 & 9 & 8 & 1 & 1 & 55 & 8 & 2 & 9 & 33 & 2 \\
\hline 1 & 1 & 11 & 16 & 1 & 1 & 57 & 8 & 2 & 9 & 39 & 8 \\
\hline 1 & 1 & 13 & 16 & 1 & 1 & 59 & 16 & 2 & 9 & 41 & 8 \\
\hline 1 & 1 & 15 & 4 & 1 & 1 & 61 & 16 & 2 & 9 & 47 & 4 \\
\hline 1 & 1 & 17 & 4 & 1 & 1 & 63 & 2 & 2 & 9 & 49 & 4 \\
\hline 1 & 1 & 19 & 16 & 4 & 3 & 1 & 1 & 2 & 9 & 55 & 8 \\
\hline 1 & 1 & 21 & 16 & 4 & 3 & 33 & 2 & 2 & 9 & 57 & 8 \\
\hline 1 & 1 & 23 & 8 & 4 & 5 & 1 & 1 & 2 & 9 & 63 & 2 \\
\hline 1 & 1 & 25 & 8 & 4 & 5 & 17 & 4 & 4 & 11 & 1 & 1 \\
\hline 1 & 1 & 27 & 16 & 4 & 5 & 33 & 2 & 4 & 11 & 33 & 2 \\
\hline 1 & 1 & 29 & 16 & 4 & 5 & 49 & 4 & 4 & 13 & 1 & 1 \\
\hline 1 & 1 & 31 & 2 & 2 & 7 & 1 & 1 & 4 & 13 & 17 & 4 \\
\hline 1 & 1 & 33 & 2 & 2 & 7 & 31 & 2 & 4 & 13 & 33 & 2 \\
\hline 1 & 1 & 35 & 16 & 2 & 7 & 33 & 2 & 4 & 13 & 49 & 4 \\
\hline 1 & 1 & 37 & 16 & 2 & 7 & 63 & 2 & 2 & 15 & 1 & 1 \\
\hline 1 & 1 & 39 & 8 & 2 & 9 & 1 & 1 & 2 & 15 & 31 & 2 \\
\hline 1 & 1 & 41 & 8 & 2 & 9 & 7 & 8 & 2 & 15 & 33 & 2 \\
\hline 1 & 1 & 43 & 16 & 2 & 9 & 9 & 8 & 2 & 15 & 63 & 2 \\
\hline 1 & 1 & 45 & 16 & 2 & 9 & 15 & 4 & & & & \\
\hline
\end{tabular}


TABLE 2 THE NUMBER OF COMPATIBLE PAIR OF ACTIONS FOR $C_{\nu_{m}} \otimes C_{2 n}$

\begin{tabular}{|c|c|c|}
\hline$m$ & $n$ & Compatible \\
\hline 4 & 1 & 15 \\
\hline 4 & 2 & 18 \\
\hline 4 & 3 & 24 \\
\hline 4 & 4 & 36 \\
\hline 4 & 5 & 52 \\
\hline 4 & 6 & 68 \\
\hline 4 & 7 & 100 \\
\hline 4 & 8 & 164 \\
\hline 4 & 9 & 292 \\
\hline \multicolumn{3}{|c|}{} \\
\hline 7 & 1 & 74 \\
\hline 7 & 2 & 80 \\
\hline 7 & 3 & 92 \\
\hline 7 & 4 & 116 \\
\hline 7 & 5 & 164 \\
\hline 7 & 6 & 260 \\
\hline 7 & 7 & 452 \\
\hline 7 & 8 & 580 \\
\hline 7 & 9 & 708 \\
\hline
\end{tabular}

\begin{tabular}{|c|c|c|}
\hline$m$ & $n$ & Compatible \\
\hline 5 & 1 & 24 \\
\hline 5 & 2 & 28 \\
\hline 5 & 3 & 36 \\
\hline 5 & 4 & 52 \\
\hline 5 & 5 & 84 \\
\hline 5 & 6 & 116 \\
\hline 5 & 7 & 148 \\
\hline 5 & 8 & 212 \\
\hline 5 & 9 & 340 \\
\hline
\end{tabular}

\begin{tabular}{|c|c|c|}
\hline$m$ & $n$ & Compatible \\
\hline 6 & 1 & 41 \\
\hline 6 & 2 & 46 \\
\hline 6 & 3 & 56 \\
\hline 6 & 4 & 76 \\
\hline 6 & 5 & 116 \\
\hline 6 & 6 & 196 \\
\hline 6 & 7 & 260 \\
\hline 6 & 8 & 324 \\
\hline 6 & 9 & 452 \\
\hline
\end{tabular}

\begin{tabular}{|c|c|c|}
\hline 8 & 1 & 139 \\
\hline 8 & 2 & 146 \\
\hline 8 & 3 & 160 \\
\hline 8 & 4 & 188 \\
\hline 8 & 5 & 244 \\
\hline 8 & 6 & 356 \\
\hline 8 & 7 & 580 \\
\hline 8 & 8 & 1028 \\
\hline 8 & 9 & 1284 \\
\hline
\end{tabular}

\begin{tabular}{|l|l|l|}
\hline 9 & 1 & 268 \\
\hline 9 & 2 & 276 \\
\hline 9 & 3 & 292 \\
\hline 9 & 4 & 324 \\
\hline 9 & 5 & 388 \\
\hline 9 & 6 & 516 \\
\hline 9 & 7 & 772 \\
\hline 9 & 8 & 1284 \\
\hline 9 & 9 & 2308 \\
\hline
\end{tabular}

Now, the results have been discovered all the cases to find the exact number of the compatible pair of actions between two finite cyclic groups of 2-power order which gives the maximum number of the non-abelian tensor product for such type groups. The order of the actions which are order one, order two and order greater than two which is $2^{s}$ where $s \geq 2$ with several cases are discovered according to the value of $t$ are considered the main point in the results in order to find the number of the compatible pair of actions. Besides that, the general formula for the number of the compatible pair of actions for the non-abelian tensor product for the finite cyclic groups of 2-power order are determined. Then some examples are given to illustrate the actions that satisfying the compatibility conditions and the number of the compatible pair of actions for such type groups.

\section{CONCLUSIONS}

The number of the compatible pair of actions are $(m-3)\left(2^{r^{\prime}-1}\right)+2^{r-1}+2^{n-1}+2^{m-1}+4$ provided $r=$ $\min \{m+1, n\}$ and $r^{\prime}=\min \{m, n\}$. This paper also gives the maximum number of different non-abelian tensor product can be found for $C_{2^{m}} \otimes C_{2^{n}}$ or $C_{2^{n}} \otimes C_{2^{m}}$. For the future research idea, the non-abelian tensor product can be determined specifically according to the number of the compatible pair of actions.

\section{ACKNOWLEDGEMENT}

The first author would like to thank Institute of Postgraduate Studies of Universiti Malaysia Pahang (UMP) for the Graduate Research Scheme (GRS).

\section{REFERENCES}

[1] Brown R. and Loday J. L. (1984). Excision homotopique en basse dimension. CR Acad. Sci. Paris SI Math. 298(15): 353-356.

[2] Brown, R., Johnson, D. L. and Robertson, E. F (1987). Some computations of non-abelian tensor products of groups. Journal of Algebra. 111: 177-202.

[3] Dummit, D. S. and Foote, R.M. (2004). Abstract Algebra. USA: John Wiley and Sons.

[4] Ellis, G. and McDermott, A. (1998). Tensor products of prime-power groups. Journal of Pure and Applied Algebra. 132(2): 119-128.

[5] Group, T. G. GAP - Groups, Algorithms and Programming. Version 6.8 (2005). http://www.gapsystem.org.Galway.

[6] Kappe, L. C. (1999). Nonabelian tensor products of groups: the commutator connection. London Mathematical Society Lecture Note Series, pp. 447-454.

[7] McDermott, A. (1998). The Nonabelian Tensor Product of Groups: Computations and Structural Results. PhD Dissertation. National University of Ireland, Galway.

[8] Mohamad M. S. (2012). Compatibility conditions and nonabelian tensor products of finite cyclic groups of $\mathrm{p}$ power order. PhD Thesis. Universiti Teknologi Malaysia.

[9] Mohamad, M. S., Sarmin, N. H., Ali, N. M. M. and L. C. Kappe (2012). The Computation of the Nonabelian Tensor Product of Cyclic Group of Order. Jurnal Teknologi, 57(1).

[10] Shahoodh, M. K., Mohamad, M. S., Yusof, Y., and Sulaiman, S. A. (2016). Compatible Pair of Nontrivial Actions for Cyclic Groups of 3-power Order. National Conference for Postgraduate Research (NCON-PGR 2016). 792-797. 
[11] Sulaiman, S. A., Mohamad, M. S., Yusof, y., Sarmin, N. H., Ali, N. M. M., Ken, T. L. and Ahmad, T. (2015). Compatible pair of nontrivial actions for some cyclic groups of 2-power order. The 2nd ISM International Statistical Conference 2014 (ISM-II): Empowering the Applications of Statistical and Mathematical Sciences. 1643:700-705.

[12] Sulaiman, S. A., Mohamad, M. S., Yusof, Y., and Shahoodh, M. K. (2016). Compatible Pair of Nontrivial Action for Finite Cyclic 2-Groups. National Conference for Postgraduate Research (NCON-PGR 2016). 39-42.

[13] Visscher M. P. (1998). The Nonabelian Tensor Product of Groups. PhD Dissertation. State University of New York. 